\title{
The curvature of the midlocus in the plane
}

\author{
Azeb Alghanemi \\ Faculty of Sciences/Department of Mathematics \\ King Abdulaziz University \\ Jeddah, Saudi Arabia \\ aalghanemi@kau.edu.sa
}

\begin{abstract}
The curvature of the midlocus associated to a smooth plane curve is investigated. Also, the impact of the type of the singularity of radius function on the relationship between the curvature of symmetry set and the associated midlocus is pointed out.
\end{abstract}

Keywords: symmetry set, midlocus, boundary, singularity.

\section{INTRODUCTION}

The concept of midlocus of a plane curve was initially introduced by Brady under the name "smoothed local symmetry" (cf. [3]). Later on the mathematical investigation of midlocus was provided by Peter Giblin and his student Brassett. In [7] they give the condition for the midlocus of a plane curve to be a regular curve. Also, they study the behaviour of the midpoint map. In [9] Peter Giblin and Warder introduce a system of ordinary differential equations generating by the midlocus of a plane curve and the radius function. The solution of the this system is the symmetry set. As a result of this, the boundary curve will be created as the envelope of the circles centered on the symmetry set. For more details on envelope we refer the reader to $[4,5,6,7,8]$. Standing on this, we can easily recognize the importance of the midlocus. The results in [9] had been generalized to the higher dimensions by the author [1]. Also, the singularities of the midlocus associated to a space curve and a surface in $\mathbb{R}^{3}$ are studied in $[1,2]$.

This paper is divided into three main parts, the first part deals with the introduction and the second part treats preliminaries which give the basic concepts of symmetry set of a plane curve and its relation with the associated midlocus. The third part, deals with the relation between the arc-length of the symmetry set of a plane curve and the associated arc-length of the midlocus and the relation between the Serret-Frenet frames of the symmetry set and the associated midlocus. Also, the curvature of the midlocus will be given in terms of the curvature of the symmetry set, the radius function and its derivatives. Moreover, the curvature of the midlocus will be given in a simple formula involving the angle between the tangent of the symmetry set and the tangent of the midlocus as well as the angle between the normal of the boundary and the tangent of the symmetry set. 


\section{PRELIMINARIES}

In this section, we review the basic concepts of a plane curve. Also, we give the essential concepts of the symmetry set of an object in the plane with smooth boundary. Also, we highlight the concept of the midlocus.

A curve $\gamma: I \rightarrow \mathbb{R}^{2}$ is a regular plane curve if $\gamma^{\prime}(t) \neq 0$ for all $t \in I$. The unit tangent vector associated to a regular plane curve $\gamma$ is defined by $T_{\gamma}=\frac{\gamma^{\prime}}{\left\|\gamma^{\prime}\right\|}$ and the associated unit normal $N_{\gamma}$ is obtained from $T_{\gamma}$ by rotating anticlockwise through a right angle. The curvature of a regular plane curve given by the parametric $\gamma(t)=\left(\gamma_{1}(t), \gamma_{2}(t)\right)$ is given by

$$
\kappa_{\gamma}=\frac{\gamma_{1}^{\prime} \gamma_{2}^{\prime \prime}-\gamma_{1}^{\prime \prime} \gamma_{2}^{\prime}}{\left(\gamma_{1}^{\prime 2}+\gamma_{2}^{\prime 2}\right)^{\frac{3}{2}}} .
$$

$\gamma$ is a unit speed curve if $\left\|\gamma^{\prime}(t)\right\|=1$ for all $t \in I$. If $\gamma$ is a unit speed plane curve, then the Serret-Frenet equations are given by

$$
\left\{\begin{array}{l}
T_{\gamma}^{\prime}=\kappa_{\gamma} N_{\gamma} \\
N_{\gamma}^{\prime}=-\kappa_{\gamma} T_{\gamma} .
\end{array}\right.
$$

In the rest of this section we present the the basic concepts of the symmetry set in the plane. Also, we highlight the relationship between the symmetry set and the boundary curve. First we give the definition of the symmetry set and the associated midlocus.

Definition 2.1 Given an object $\Omega$ in $\mathbb{R}^{2}$ with smooth boundary curve $\gamma_{b}$ the symmetry set $S$ is the clousure of the locus of centres of circles, bitangent to $\gamma_{b}$. The midlocus associated to $\gamma_{b}$ is the locus of the midpoints of chords joining the tangency points.

Now if $\gamma$ is the regular part of the symmetry set and $x_{0}$ be a point on $\gamma$. If $x_{1}$ and $x_{2}$ are the associated boundary (tangency) points, then the associated boundary curves around $x_{1}$ and $x_{2}$ are given by $($ cf. $[1,6,8])$

$$
\gamma_{i}=\gamma-r^{\prime} r T_{\gamma} \pm r \sqrt{1-r^{\prime 2}} N_{\gamma}, i=1,2
$$

where $T_{\gamma}$ (resp. $N_{\gamma}$ ) is the unit tangent (resp. unit normal) of $\gamma, r$ is the radius function and prime denotes the derivative with respect to the arc-length of $\gamma$.

In this case $\gamma_{1}$ and $\gamma_{2}$ are oriented in opposite direction and their unit normals are pointed to the center of the bitangent circle as show in figure 1 .

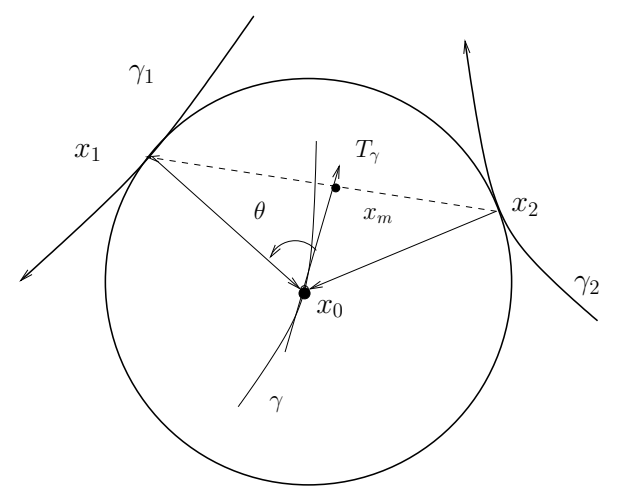

Figure 1: The symmetry point $x_{0}$ and the associated midlocus $x_{m}$. 
Also, the associated midlocus is given by

$$
\gamma_{m}=\frac{\gamma_{1}+\gamma_{2}}{2}=\gamma-r^{\prime} r T_{\gamma}
$$

The unit tangents and the unit normal of the boundary at the tangency points are given by

Lemma $2.2[8]$ Let $\gamma$ be the symmetry set of a smooth plane curve $\gamma_{b}$ and $r$ be the radius function. The unit tangents $T_{1}$ and $T_{2}$ and unit normals $N_{1}$ and $N_{2}$ of $\gamma_{b}$ at the tangency points associated to a smooth point $x_{0} \in \gamma$ are given by

$$
\left\{\begin{array}{l}
T_{1}=-\sqrt{1-r^{\prime 2}} T_{\gamma}-r^{\prime} N_{\gamma} \\
T_{2}=\sqrt{1-r^{\prime 2}} T_{\gamma}-r^{\prime} N_{\gamma} \\
N_{1}=r^{\prime} T_{\gamma}-\sqrt{1-r^{\prime 2}} N_{\gamma} \\
N_{2}=r^{\prime} T_{\gamma}+\sqrt{1-r^{\prime 2}} N_{\gamma}
\end{array}\right.
$$

where, $T_{\gamma}$ and $N_{\gamma}$ are the unit tangent and unit normal of the symmetry set at $x_{0}$ and prime denotes the derivative with respect to the arc-length of the symmetry set.

\section{CURVATURE OF THE MIDLOCUS ASSOCIATED TO A PLANE CURVE}

In this section we are going to study the curvature of the midlocus associated to smooth symmetry set of an object in $\mathbb{R}^{2}$ with smooth boundary (for the applications of symmetry set and related medial axis we refer reader to [10] ). In this section we assume that the midlocus is a regular curve. Let $\gamma$ be the symmetry set of a plane object $\Omega$ with smooth boundary parametrized by its arc-length $s$, then the associated midlocus is given by $\gamma_{m}=\gamma-r r^{\prime} T_{\gamma}$, where $T_{\gamma}$ is the unit tangent of $\gamma$ and prime is the derivative with respect to the arc-length $s$. By direct calculation we easily obtain the following lemma.

Lemma 3.1 Let $\gamma$ be the smooth part of the symmetry set of an object $\Omega$ in $\mathbb{R}^{2}$ with smooth boundary $\gamma_{b}$ and $r$ be the radius function. If $\gamma_{m}$ is the smooth midlocus associated to $\gamma$. Then,

$$
\begin{aligned}
& \text { 1. } \frac{d s_{m}}{d s}=\sqrt{\left(1-r^{\prime 2}-r r^{\prime \prime}\right)^{2}+r^{2} r^{\prime 2} \kappa_{\gamma}^{2}}, \\
& \text { 2. } T_{\gamma_{m}}=\frac{1-r^{\prime 2}-r r^{\prime \prime}}{\sqrt{\left(1-r^{\prime 2}-r r^{\prime \prime}\right)^{2}+r^{2}{r^{\prime}}^{2} \kappa_{\gamma}^{2}}} T_{\gamma}-\frac{r r^{\prime} \kappa_{\gamma}}{\sqrt{\left(1-r^{\prime 2}-r r^{\prime \prime}\right)^{2}+r^{2}{r^{\prime}}^{2} \kappa_{\gamma}^{2}}} N_{\gamma}, \\
& \text { 3. } N_{\gamma_{m}}=\frac{r r^{\prime} \kappa_{\gamma}}{\sqrt{\left(1-r^{\prime 2}-r r^{\prime \prime}\right)^{2}+r^{2} r^{\prime 2} \kappa_{\gamma}^{2}}} T_{\gamma}+\frac{1-r^{\prime 2}-r r^{\prime \prime}}{\sqrt{\left(1-r^{\prime 2}-r r^{\prime \prime}\right)^{2}+r^{2} r^{\prime 2} \kappa_{\gamma}^{2}}} N_{\gamma} .
\end{aligned}
$$

where, $\kappa_{\gamma}$ is the curvature of $\gamma, s\left(\right.$ resp. $\left.s_{m}\right)$ is the arc-length of $\gamma\left(\right.$ resp. $\left.\gamma_{m}\right)$ and prime denotes the derivative with respect to the arc-length of $\gamma$.

Remark 3.2 It is clear form part 2 in Lemma 3.1 to observe that the tangents of the symmetry set and the associated midlocus are parallel if and only if $r^{\prime}=0$ or $\kappa_{\gamma}=0$. If $r^{\prime}=0$, then the tangents of the boundary curve at the tangency points are parallel. Moreover, the symmetry point and the associated midlocus point are coincide. If $\kappa_{\gamma}=0$, then the curvatures of the boundary at the tangency points are equal (this can be obtained directly from proposition 5.2.9 in [1]).

Now we use Lemma 3.1 to calculate the curvature of $\gamma_{m}$. Put $T_{\gamma_{m}}=\alpha T_{\gamma}+\beta N_{\gamma}$, where $\alpha$ and $\beta$ are given by 2 in Lemma 3.1. Differentiate with respect to the arc-length of $\gamma$ we obtain

$$
\kappa_{\gamma_{m}} N_{\gamma_{m}} \frac{d s_{m}}{d s}=\left(\alpha^{\prime}-\beta \kappa_{\gamma}\right) T_{\gamma}+\left(\alpha \kappa_{\gamma}+\beta^{\prime}\right) N_{\gamma} .
$$


Now we have

$$
\begin{aligned}
\kappa_{\gamma_{m}} N_{\gamma_{m}} \cdot N_{\gamma_{m}} \frac{d s_{m}}{d s} & =\kappa_{\gamma_{m}} \frac{d s_{m}}{d s} \\
& =-\beta\left(\alpha^{\prime}-\kappa_{\gamma} \beta\right)+\alpha\left(\kappa_{\gamma} \alpha+\beta^{\prime}\right) \\
& =-\alpha^{\prime} \beta+\alpha \beta^{\prime}+\kappa_{\gamma}\left(\alpha^{2}+\beta^{2}\right) \\
& =-\alpha^{\prime} \beta+\alpha \beta^{\prime}+\kappa_{\gamma} \\
& =\alpha^{2}\left(\frac{\beta}{\alpha}\right)^{\prime}+\kappa_{\gamma} .
\end{aligned}
$$

But we have $\frac{\beta}{\alpha}=\frac{-r r^{\prime} \kappa_{\gamma}}{1-r^{\prime 2}-r r^{\prime \prime}}$. Therefore,

$$
\alpha^{2}\left(\frac{\beta}{\alpha}\right)^{\prime}=\frac{-\left(1-r^{\prime 2}-r r^{\prime \prime}\right)\left(r^{\prime 2} \kappa_{\gamma}+r r^{\prime \prime} \kappa_{\gamma}+r r^{\prime} \kappa_{\gamma}^{\prime}\right)-r^{2} r^{\prime} \kappa_{\gamma}\left(3 r^{\prime \prime}+r^{\prime \prime \prime}\right)}{\left(1-r^{\prime 2}-r r^{\prime \prime}\right)^{2}+r^{2} r^{\prime 2} \kappa_{\gamma}^{2}} .
$$

By substituting in equation (3) we get

$\kappa_{\gamma_{m}} \frac{d s_{m}}{d s}=\frac{\kappa_{\gamma}\left[\left(1-{r^{\prime}}^{2}-r r^{\prime \prime}\right)^{2}+r^{2}{r^{\prime}}^{2} \kappa_{\gamma}^{2}\right]-\left(1-{r^{\prime}}^{2}-r r^{\prime \prime}\right)\left({r^{\prime}}^{2} \kappa_{\gamma}+r r^{\prime \prime} \kappa_{\gamma}+r r^{\prime} \kappa_{\gamma}^{\prime}\right)-r^{2} r^{\prime} \kappa_{\gamma}\left(3 r^{\prime \prime}+r^{\prime \prime \prime}\right)}{\left(1-{r^{\prime}}^{2}-r r^{\prime \prime}\right)^{2}+r^{2}{r^{\prime}}^{2} \kappa_{\gamma}^{2}}$.

Now using Lemma 3.1 we obtain the following.

Theorem 3.3 Let $\gamma$ be the smooth part of the symmetry set of an object $\Omega$ in $\mathbb{R}^{2}$ with smooth boundary $\gamma_{b}$ and $\gamma_{m}$ be the associated smooth midlocus. Then the curvature of the midlocus is given by

$$
\kappa_{\gamma_{m}}=\frac{\kappa_{\gamma}\left[\left(1-{r^{\prime}}^{2}-r r^{\prime \prime}\right)^{2}+r^{2}{r^{\prime 2}}^{2} \kappa_{\gamma}^{2}\right]-\left(1-{r^{\prime 2}}^{2}-r r^{\prime \prime}\right)\left(r^{\prime 2} \kappa_{\gamma}+r r^{\prime \prime} \kappa_{\gamma}+r r^{\prime} \kappa_{\gamma}^{\prime}\right)-r^{2} r^{\prime} \kappa_{\gamma}\left(3 r^{\prime \prime}+r^{\prime \prime \prime}\right)}{\left[\left(1-r^{\prime 2}-r r^{\prime \prime}\right)^{2}+r^{2}{r^{\prime}}^{2} \kappa_{\gamma}^{2}\right]^{\frac{3}{2}}},
$$

where $\kappa_{\gamma}$ is the curvature of $\gamma$ and prime is the derivative with respect to the arc-length of $\gamma$.

Definition 3.4 A smooth function $f: \mathbb{R} \rightarrow \mathbb{R}$ is said to have an $A_{k}$ singularity at $t_{0}$ if and only if $f^{\prime}\left(t_{0}\right)=f^{\prime \prime}\left(t_{0}\right)=\ldots=f^{k}\left(t_{0}\right)=0$ and $f^{k+1}\left(t_{0}\right) \neq 0$.

The following corollary can be easily obtained from Theorem 3.3.

Corollary 3.5 Assume as in Theorem 3.3.

1. If the radius function has an $A_{1}$ singularity at $s_{0}$ i.e. $r^{\prime}\left(s_{0}\right)=0, r^{\prime \prime}\left(s_{0}\right) \neq 0$. Then, $\kappa_{\gamma_{m}}=$ $\delta\left(\frac{\kappa_{\gamma}\left(1-2 r r^{\prime \prime}\right)}{\left(1-r r^{\prime \prime}\right)^{2}}\right)$, where $\delta$ is the sign of $\left(1-r r^{\prime \prime}\right)$.

2. If the radius function has an $A_{k \geqslant 2}$ singularity at $s_{0}$, i.e. $r^{\prime}\left(s_{0}\right)=r^{\prime \prime}\left(s_{0}\right)=0$. Then, $\kappa_{\gamma_{m}}=\kappa_{\gamma}$.

Although equation (4) is useful for studying the relationship between the radius function and the curvature of the midlocus, it is very complicated. Our task now is to find a simple formula for the curvature of the midlocus. The following theorem gives such a formula.

Theorem 3.6 Let $\gamma$ be the smooth part of the symmetry set of an object $\Omega$ in $\mathbb{R}^{2}$ with smooth boundary $\gamma_{b}$ and $\gamma_{m}$ be the associated smooth midlocus. Let $\theta$ be the angle between the normal of the boundary and the tangent of the symmetry set and $\phi$ is the angle between the tangent of the symmetry set and the tangent of the midlocus. The curvature of the midlocus is given by

$$
\kappa_{\gamma_{m}}=\frac{\cos \phi\left(\kappa_{\gamma}+\phi^{\prime}\right)}{\sin \theta\left(\sin \theta+r \theta^{\prime}\right)},
$$

where $\kappa_{\gamma}$ is the curvature of $\gamma$ and prime is the derivative with respect to the arc-length of $\gamma$. 
Proof: Let $\gamma$ be the regular part of the symmetry set of an object $\Omega$ in $\mathbb{R}^{2}$ parametrized by its arc-length with smooth boundary. Let $\gamma_{1}$ and $\gamma_{2}$ be two smooth pieces of the boundary curve around the tangency points as shown in figure 1. Let $\theta$ be the angle between the tangent of the symmetry set and the normal of the boundary as shown in figure 1. From Lemma 2.2 it is clear that $\cos \theta=r^{\prime}$. Now if $\phi$ is the angle between the tangent of the symmetry set and the tangent of the associated midlocus $T_{\gamma_{m}}$, then

$$
T_{\gamma_{m}}=\cos \phi T_{\gamma}+\sin \phi N_{\gamma} .
$$

Using equation (6) and equation (3), we obtain that

$$
\begin{aligned}
\kappa_{\gamma_{m}} \frac{d s_{m}}{d s} & =\cos ^{2} \phi(\tan \phi)^{\prime}+\kappa_{\gamma} . \\
& =\phi^{\prime}+\kappa_{\gamma}
\end{aligned}
$$

Therefore,

$$
\kappa_{\gamma_{m}}=\frac{\phi^{\prime}+\kappa_{\gamma}}{\frac{d s_{m}}{d s}} .
$$

From Lemmas 2.2 and 3.1 it can be shown that

$$
\frac{d s_{m}}{d s}=\frac{\sin \theta\left(\sin \theta+r \theta^{\prime}\right)}{\cos \phi}
$$

Therefore,

$$
\kappa_{\gamma_{m}}=\frac{\cos \phi\left(\kappa_{\gamma}+\phi^{\prime}\right)}{\sin \theta\left(\sin \theta+r \theta^{\prime}\right)}
$$

which completes the proof.

\section{CONCLUSION}

In this paper we present the curvature of the midlocus of an object in the plane with smooth boundary in two methods. We hope this will find implementations in shape recognition, shape reconstruction, medical imaging, and describing objects.

\section{ACKNOWLEDGEMENTS}

The author is grateful to professor Peter Giblin and Dr. Kevin Houston for useful comments and conversation.

\section{References}

[1] Azeb Alghanemi. Geometry of skeletal structures and symmetry sets. Ph.D. Thesis University of Leeds, 2012.

[2] Azeb Alghanemi and Peter Giblin. On geometry of the midlocus associated to a smooth curve in plane and space, submitted https://www.liverpool.ac.uk/ pjgiblin/. 
[3] M. Brady and H. Asada. Smoothed local symmetries and thier implementation. International Journal of Robotics Research, 3:36 - 61, 1984.

[4] J. W. Bruce and P. J. Giblin. Growth, motion and one parameter families of symmetry sets. Proceedings of the Royal Society of Edinburgh Sect. A, 104:179-204, 1986.

[5] J. W. Bruce and P. J. Giblin. Curves and singularities. Cambridge University Press, 1992.

[6] J. W. Bruce, P. J. Giblin, and C. G. Gibson. Symmetry sets. Proc.Roy. Soc. Edinburgh Sect. A, 101:163-186, 1985.

[7] P. J. Giblin and S. A. Brassett. Local symmetry of plane curves. Amer. Math. Monthly, 92:689- 707, 1985.

[8] P. J. Giblin and B. B. Kimia. On the intrinsic reconstructions of shape from its symmetries. IEEE Transactions on Pattern, Analysis and Machine Intelligence, 25:895-911, 2003.

[9] P. J. Giblin and J. P. Warder. Reconstruction from medial representations. Amer. Math. Monthly, 118:712- 725, 2011.

[10] K. Siddiqi and M. Pizer. Medial representations: mathematics, algorithms and applications. Springer ISBN 978-1-4020-8657-1, 2008.

\section{AUTHOR'S BIOGRAPHY:}

Full Name: Dr. Azeb Alghanemi.

Title: Assistant Professor of Mathematics.

Institution: King Abdulaziz University, Jeddah Saudi Arabia.

Qualifications: B.Sc.(Mathematics- King Abdulaziz University with first honor) M.Sc.(MathematicsKing Abdulaziz University) Ph.D.(Mathematics- The University of Leeds, U.K).

Research Interests: Singularity Theory and its applications to differential geometry. Symmetry set, medial axis, skeletal structures and their applications. Riemannian geometry.

Professional experience: I worked as a demonstrator at King Abdulaziz University, Department of Mathematics from 2003 until 2012. Now I am an assistant professor at King Abdulaziz University, Department of Mathematics. 